\title{
Chest Drainage
}

\author{
Shaunagh McDermott, FFR RCSI ${ }^{1} \quad$ Diane A. Levis, PA-C ${ }^{1}$ Ronald S. Arellano, MD ${ }^{1}$ \\ ${ }^{1}$ Division of Abdominal Imaging and Interventional Radiology, \\ Department of Radiology, Massachusetts General Hospital, Boston, \\ Massachusetts

\begin{abstract}
Address for correspondence and reprint requests Shaunagh Radiology, Department of Radiology, Massachusetts General Hospital, Boston, MA 02114 (e-mail: smcdermott1@partners.org).
\end{abstract} \\ McDermott, FFR RCSI, Division of Abdominal Imaging and Interventional
}

Semin Intervent Radiol 2012;29:247-255
Abstract
Keywords
- pneumothorax
- empyema
- pleural effusion
- abscess
- thoracentesis
- thoracostomy

Infectious, traumatic, or neoplastic processes in the chest often result in fluid collections within the pleural, parenchymal, or mediastinal spaces. The same fundamental principles that guide drainages of the abdomen can be applied to the chest. This review discusses various pathologic conditions of the thorax that can result in the abnormal accumulation of fluid or air, and their management using image-guided methods.
Objectives: Upon completion of this article, the reader will be able to discuss the different interventional procedures in the chest and their indications.

Accreditation: Tufts University School of Medicine is accredited by the Accreditation Council for Continuing Medical Education to provide continuing medical education for physicians.

Credit: Tufts University School of Medicine designates this journal-based CME activity for a maximum of 1 AMA PRA Category 1 Credit $^{\mathrm{TM}}$. Physicians should claim only the credit commensurate with the extent of their participation in the activity.

Chest drainage procedures can be classified based on the anatomical regions of the pleural space, lung parenchyma, and mediastinum. This article reviews the indications, techniques (including adjunctive procedures), and management of imageguided percutaneous drainage procedures of the chest.

\section{The Pleural Space: Pathologic Conditions}

Infectious, inflammatory, hemorrhagic, and iatrogenic processes can all result in pleural space-based abnormalities.

\section{Pneumothorax}

Pneumothorax is an imaging finding and clinical condition in which air accumulates within the pleural space. Pneumothoraces can be either spontaneous, associated with under- lying lung disease (lymphangioleiomyomatosis, blebs, etc.), or the result of traumatic injury to the chest wall, pulmonary parenchyma, or airways. Traumatic pneumothorax most often results from penetrating or blunt trauma, or as an iatrogenic complication of thoracentesis, central venous catheterization, or lung biopsy (transbronchial or transthoracic). Spontaneous pneumothorax may be divided into a primary form, which has no identifiable cause and is often related to rupture of an apical intrapleural bleb, and a secondary form, which is associated with underlying parenchymal lung disease. The clinical results of pneumothoraces can range from inconsequential, to chest pain and decreased oxygenation, to tension pneumothorax that requires immediate attention.

\section{Pleural Effusion}

Pleural effusion is defined as accumulation of fluid in the pleural space. The pleural space is normally filled with $\sim 5$ to $10 \mathrm{~mL}$ of serous fluid. Intrapleural pressure is lower than the interstitial fluid pressure of the pleural tissues, favoring flow of fluid into the pleural space. The protein and cellular concentration in this fluid is typically low because pleural fluid is effectively a filtrate. ${ }^{1}$ Normally, the influx of fluid into the pleural space is balanced by its removal via the lymphatic system. In certain clinical conditions, the balance between the secretion and absorption can be disturbed and fluid may accumulate in the pleural space. In disease states, the normal composition of pleural fluid is altered, which allows for diagnosis via pleural fluid analysis.
Issue Theme Biopsies and Drains; Guest Editor, Jonathan M. Lorenz, MD, FSIR
Copyright (c) 2012 by Thieme Medical Publishers, Inc., 333 Seventh Avenue, New York, NY 10001, USA.

Tel: +1(212) 584-4662.
DOI http://dx.doi.org/ $10.1055 / \mathrm{s}-0032-1330058$. ISSN 0739-9529. 
Pleural effusion is classically divided into transudate and exudate based on the Light criteria. ${ }^{2}$ The Light criteria consist of measurement of the lactate dehydrogenase (LDH) and protein concentration in the pleural fluid and serum; fluid is considered exudative if one of the following criteria are present:

- Pleural fluid-to-serum protein ratio $>0.5$, or

- Pleural fluid-to-serum LDH ratio $>0.6$, or

- Pleural fluid LDH concentration $>200 \mathrm{U} / \mathrm{L}$ (later changed to two-thirds upper limit of normal for serum LDH)

The original study by Light evaluated 150 patients with pleural effusions, of whom only two were misclassified, which resulted in a sensitivity of $99 \%$ and a specificity of $98 \%$ for identifying exudates. ${ }^{2}$ Subsequent studies confirmed the high sensitivity of the Light criteria, but reported a lower specificity of 65 to $85 \%{ }^{3}$ A meta-analysis by Heffner showed good sensitivity and specificity of alternative diagnostic test strategies that are based solely on pleural fluid tests. The twotest and three-test rules require a single criterion to be met to diagnose an exudate. With the two-test rule, one of the following must be met to indicate the presence of an exudate:

- Pleural fluid cholesterol $>45 \mathrm{mg} / \mathrm{dL}$, or

- Pleural fluid LDH $>0.45$ of upper limit of normal serum LDH.

The three-test rule, which also requires one of the criterion to be met to make the diagnosis of an exudate, includes the preceding two criteria and also pleural fluid protein $>2.9 \mathrm{~g} / \mathrm{dL}$.

Regardless of which set of criteria is used, further analysis is required if the fluid is determined to be an exudate.

Transudates occur as an ultrafiltration of serum across pleural membranes and results from imbalance of hydrostatic or osmotic pressure. However, in exudates, the capillary beds themselves are diseased, and the increased permeability results in fluid leaking into the pleural space. - Table 1 outlines the causes of pleural effusions.

Management of patients with pleural effusions depends on the suspected disease process, the type of effusion, and the patient's symptoms. Treatment can be thought of in terms of either treatment of the underlying disease, or conservative versus invasive treatment of the effusion itself.

\section{Empyema}

Empyema is a collection of purulent fluid in the pleural space. The most common cause is pneumonia. Lung abscess, bronchopleural fistula, esophageal perforation, postsurgical complications, and trauma may also result in empyema. There are three stages in the evolution of empyema: exudative stage, with a small amount of sterile fluid in the pleural space; fibropurulent stage, where fluid tends to be loculated; and organized phase, where a thick pleural peel prevents the lung from reexpanding. ${ }^{4}$

Along with antibiotic therapy and treatment of the underlying disease process, early and complete drainage of the infected fluid is considered essential in the successful management of empyema.
Table 1 Causes of Pleural Effusions

\begin{tabular}{|c|c|}
\hline Transudate & $\begin{array}{l}\text { Congestive heart failure } \\
\text { Nephrotic syndrome } \\
\text { Liver cirrhosis } \\
\text { Hypoalbuminemia }\end{array}$ \\
\hline \multirow[t]{6}{*}{ Exudate } & $\begin{array}{l}\text { Infectious } \\
\text { - Parapneumonic } \\
\text { - Tuberculous pleurisy }\end{array}$ \\
\hline & Pulmonary embolism \\
\hline & Malignancy \\
\hline & $\begin{array}{l}\text { Drug induced } \\
\text { - Amiodarone } \\
\text { - Nitrofurantoin } \\
\text { - Methotrexate } \\
\text { - Phenytoin }\end{array}$ \\
\hline & $\begin{array}{l}\text { Connective tissue disease } \\
\text { - Lupus pleuritis } \\
\text { - Rheumatoid arthritis }\end{array}$ \\
\hline & $\begin{array}{l}\text { Other } \\
\text { - Pancreatitis } \\
\text { - Esophageal perforation } \\
\text { - Postcoronary surgery } \\
\text { - Ovarian hyperstimulation syndrome } \\
\text { - Meigs syndrome } \\
\text { - Dressler syndrome } \\
\text { - Thoracic duct disruption }\end{array}$ \\
\hline
\end{tabular}

\section{Pleural Space Intervention}

The different procedures involving the pleural space include thoracentesis, chest drain insertion, tunneled catheter insertion, and pleurodesis.

\section{Thoracentesis}

An estimated 178,000 thoracenteses are performed among 1.5 million patients with pleural effusion each year in the United States. ${ }^{5}$ Although the procedure can be performed at bedside without imaging guidance, it is generally recommended to use ultrasonographic guidance to avoid potential complications. Continuous ultrasound guidance reduces the risk of iatrogenic pneumothoraces compared with nonguided thoracenteses, with reported reductions from 10 to $29 \%$ without guidance, to 0 to $5 \%$, with ultrasound guidance. ${ }^{6-8}$ The use of ultrasound has also been shown to reduce the likelihood of hemorrhage by 38.7\%. ${ }^{9}$ Between 1993 and 2008, the share of thoracentesis performed by radiologists and pulmonary and critical care medicine physicians changed from $10 \%$ and $49 \%$ to $52 \%$ and $27 \%{ }^{10}$ 
Complications of thoracentesis include pneumothorax, hemothorax, reexpansion pulmonary edema (RPE), and organ laceration. Other complications include those involving the skin and subcutaneous tissue, such as pain, hematoma/ hemorrhage and infection, and injury to the neurovascular bundle. Pneumothorax is the most common major complication of thoracentesis, with a recent meta-analysis quoting a $6 \%$ pneumothorax rate with $34 \%$ of pneumothoraces requiring chest drain insertion. ${ }^{11}$ In some patients with chronic pleural effusions and underlying lung parenchymal disease, pneumothoraces can occur due to the inability of the lung to fully reexpand ${ }^{11,12}$ (- Fig. 1). In these circumstances, the radiographic appearance of a pneumothorax is usually not the result of air leak but of the unexpanded lung. These pneumothoraces (which the authors term vacu-thorax) either resolve slowly or not at all but are not associated with respiratory distress or progression, and attempts at evacuation with chest tubes are unsuccessful in most cases.

Reexpansion pulmonary edema is a potentially life-threatening complication after thoracentesis or chest drain insertion (-Fig. 2). The incidence of RPE has been reported to be anywhere from 0.2 to $14 \%,{ }^{13}$ and it may or may not be related to the total amount of fluid removed. An arbitrary threshold for fluid removal of $1 \mathrm{~L}$ has been suggested by some to prevent RPE developing, ${ }^{14}$ although this is controversial. Others have found that clinical and radiographic RPE after large volume thoracentesis is rare and independent of the volume of fluid removed and that large effusions can, and should, be drained completely as long as chest discomfort does not develop. ${ }^{13}$

Hemorrhagic complications related to thoracentesis usually result from injury to the intercostal artery ${ }^{15,16}$ (- Fig. 3). Traditional anatomical teaching suggests that the intercostal artery lies within the costal groove, which is located along the inferior portion of the rib. However, a recent study reviewing the location of the posterior intercostal artery on computed tomography (CT) found considerable variability in the position and tortuosity of the vessel, which only comes to lie within the subcostal groove toward the midaxillary line. ${ }^{17}$ They advised additional caution to avoid the posterior paravertebral area, where the artery was found to lie halfway between the two ribs in the intercostal space. The same conclusions were made from a separate study using threedimensional CT angiography. ${ }^{18}$

The risk of infection after thoracentesis is extremely low, with one study finding no evidence of infection after 2489 ultrasound-guided thoracenteses. ${ }^{19}$

\section{Tunneled Pleural Catheter Insertion}

Tunneled pleural catheters (TPCs) have become a popular therapeutic tool in the management of chronic pleural effusions. Although the main indication for a TPC has been recurrent, symptomatic, malignant pleural effusion, there are increasing reports of TPC use for other medical conditions. A recent systematic review of 19 studies with a total of 1370 patients found that symptomatic improvement was reported in $95.6 \%{ }^{20}$ This study also found that the risk of serious complications was rare, with reported rates of empyema in $2.8 \%$, pneumothorax requiring chest drain in $5.9 \%$, and unspecified pneumothorax in $3.9 \%$.

\section{Chest Drain Insertion}

Indications for chest drain insertion include pneumothorax, hemothorax, pleural effusion, and empyema. Ultrasonography, CT, fluoroscopy, or any combination of these techniques may accurately guide chest drain placement. Image guidance is selected by (1) availability and convenience of the various techniques, (2) size and position of the collection, (3) the patient's condition, and (4) the radiologist's preference.

Ultrasonography is usually the technique of choice to guide pleural drainage. Its advantages include absence of ionizing radiation, portability, and real-time capabilities. Its portability allows bedside chest drain insertion in critically ill and hemodynamically unstable patients. CT-guided thoracostomy is beneficial for drainage of loculated pleural collections, usually associated with underlying parenchymal consolidation; it allows the radiologist to select a safe drainage pathway into the collection. Fluoroscopy can be used to access large collections, and continuous real-time monitoring of the course of the needle, guidewire, and catheter is a distinct advantage of fluoroscopy.
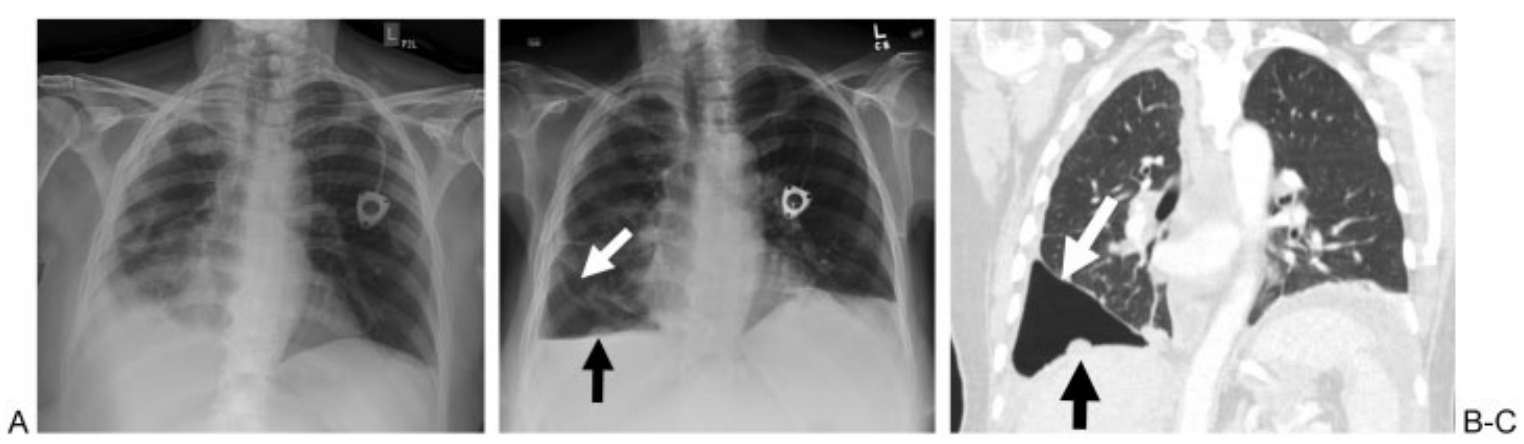

Figure 1 A 65-year-old woman with metastatic ovarian cancer. (A) Frontal radiograph of the chest demonstrates a right pleural effusion. (B) Frontal radiograph of the chest demonstrates a new right hydropneumothorax immediately after right thoracentesis (white arrow). A pleuralbased nodule is also noted (black arrow). (C) Coronal computed tomography scan of the chest obtained 1 day after the thoracentesis demonstrates incomplete expansion of the right lower lobe (white arrow), consistent with so-called vacu-thorax. Again demonstrated is the pleural-based metastasis (black arrow). The patient remained asymptomatic throughout her clinical course and did not have any further intervention. 

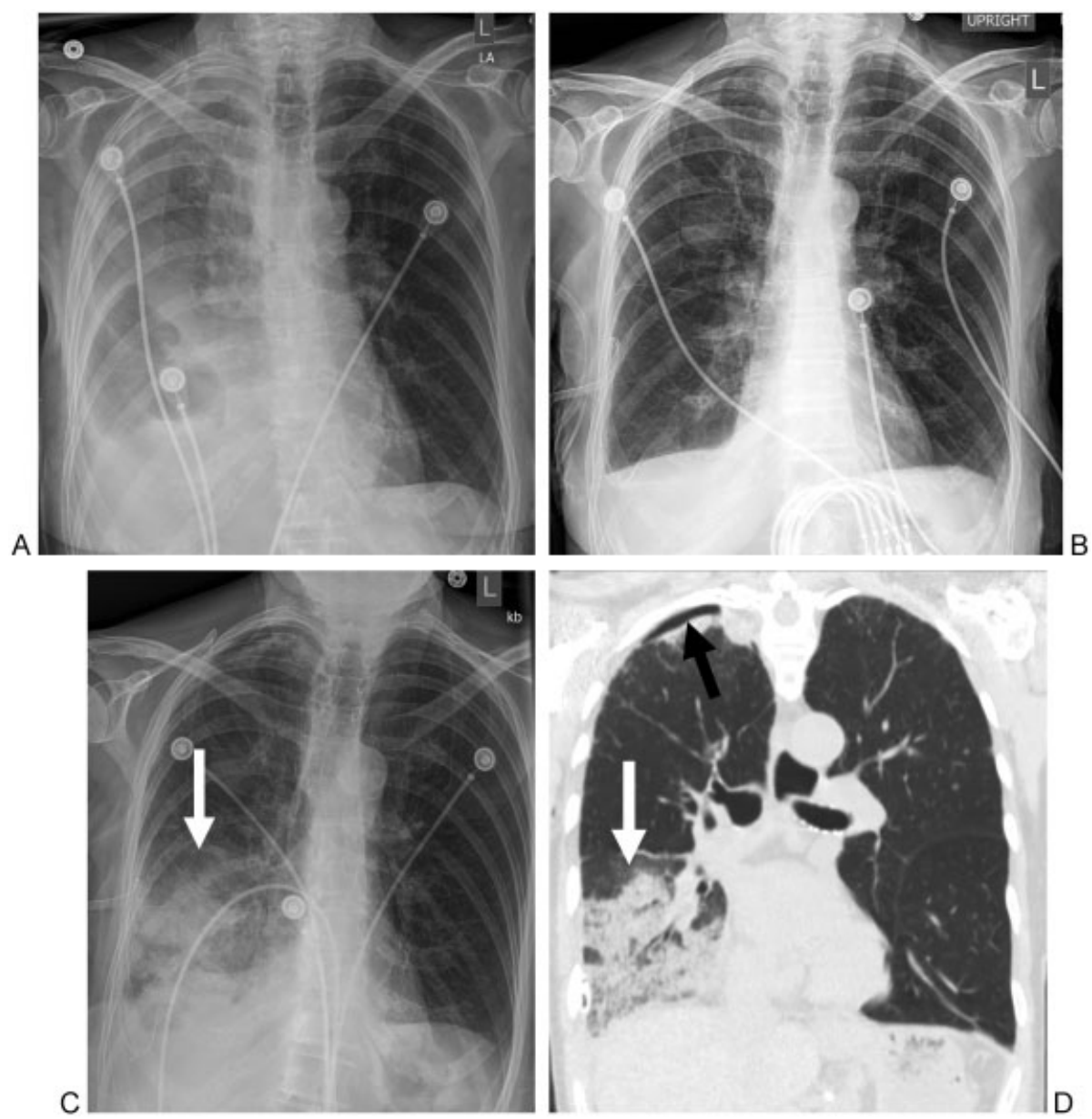

Figure 2 An 88-year-old woman with a history of a recent myocardial infarction. (A) Frontal radiograph of the chest demonstrates a right pleural effusion. (B) Frontal radiograph of the chest immediately after chest drainage shows resolution of the right pleural effusion. (C) Frontal radiograph of the chest obtained 5 hours after chest drainage and obtained for new shortness of breath demonstrates new right lower lobe airspace opacities (arrow). (D) Coronal computed tomography scan of the chest demonstrates right lower lobe airspace disease (white arrow) consistent with reexpansion pulmonary edema. There is also a small right apical pneumothorax (black arrow).

Chest drain insertion can be done using either the trocar or Seldinger technique. Proponents of the trocar method, such as Silverman et al, ${ }^{21}$ believe that the trocar method is superior to the Seldinger technique. According to these researchers, the use of exchange guidewires and dilators in conjunction with the Seldinger technique may allow introduction of air, increasing the likelihood of pneumothorax. Furthermore, these researchers contend that it is difficult to advance a catheter through the intercostal space and thickened pleura due to buckling the guidewire or catheter and that such kinking can result in the loss of access or leakage of pleural contents along the dilatation path. Proponents of the
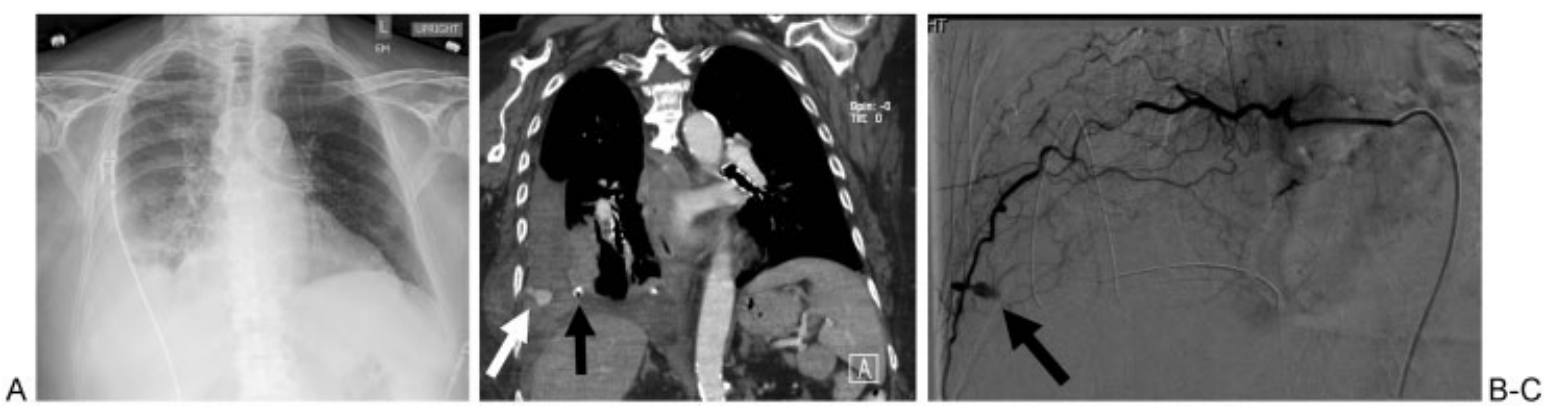

Figure 3 A 68-year-old woman with end-stage renal disease. (A) Frontal radiograph of the chest demonstrates a large right pleural effusion prior to thoracentesis. (B) Coronal computed tomography of the chest demonstrates a pseudoaneurysm of a right intercostal artery (white arrow), adjacent to a drainage catheter (black arrow) that was inserted because of shortness of breath post thoracentesis. (C) Digital subtraction angiogram demonstrates a pseudoaneurysm of a right intercostal artery (black arrow), which was successfully embolized with coils. 
Seldinger technique argue that placement of the chest tube over a guidewire allows more control and decreases the likelihood of complications. Ultimately, the technique with which the operator is most comfortable should be used.

Traditionally, large bore $(>28 \mathrm{~F})$ catheters were recommended in almost all situations that required chest drainages. However, this requires a moderately large skin incision, typically using blunt dissection and blind insertion. However, in recent years there has been a global trend toward increased use of small-bore chest drains ( 8 to $16 \mathrm{~F}$ ). The small drains have several advantages: They are easier and less painful to insert, better tolerated once placed, and have lower placement-related complication rates. In one summary of chest drain complications, ${ }^{22}$ the incidence of injury with large-bore versus small-bore tubes was $1.4 \%$ versus $0.2 \%$, the incidence of malposition was $6.5 \%$ versus $0.6 \%$, and the incidence of empyema was $1.4 \%$ versus $0.2 \%$, respectively. The one potential disadvantage, however, is a slightly increased incidence of drain blockage with smaller tubes (8.1\%) versus large-bore catheters (5.2\%).

At the authors' institution, the interventional radiology service takes "ownership" of chest tubes and drainage catheters and works collaboratively with the medical and surgical services in the day-to-day management of the catheters. This requires daily flushes with small aliquots of normal saline to maintain catheter patency as well as evaluation of daily catheter outputs. The decision to remove a chest tube is based both on radiologic findings on chest X-ray and clinical parameters (e.g., daily output, persistence or absence of air leak). A suggested algorithm for the management of chest tubes is shown in - Fig. 4. Although most chest drain-related complications occur at the time of placement, complications such as hemo-and pneumothoraces can also occur at the time of removal $^{22,23}$ (- Fig. 5). The rate of recurrence of pneumothorax after chest drain removal ranges from $2 \%$ to $24 \%$ with a reinsertion rate of 1 to $6 \%{ }^{23}$ The authors routinely perform a chest X-ray after chest tube removal; however, recent studies have found it safe not to do so in selected patients. ${ }^{24,25}$

Several options exist if clinical and radiographic assessment determines that drainage is inadequate. Occasionally, the indwelling chest tube requires repositioning to enhance the drainage of loculated collections. Also, conversion to a catheter with a larger lumen may promote adequate drainage of thick pus or bloody material. Intrapleural administration of fibrinolytic medication may aid in septated collections or collections with multiple locules (-Fig. 6). Although singlecenter studies have found the use of intrapleural fibrinolytic therapy to be effective in improving drainage of loculated effusions not drained by catheters alone, ${ }^{26-29}$ a meta-analysis $^{30}$ and a double-blind clinical trial in 454 patients ${ }^{31}$ concluded that the intrapleural administration of fibrinolytic therapy did not improve mortality or surgery rates. Conversely, a more recent study has found that the combination of intrapleural tissue plasminogen activator (tPA) and DNase improved fluid drainage in patients with pleural infection and reduced the frequency of surgical referral and the duration of the hospital stay. The study also concluded that treatment with either DNase alone or tPA alone was ineffective. ${ }^{32}$

\section{The Lung Parenchyma: Pathologic Conditions}

The most common indication for draining the lung parenchyma is a lung abscess.

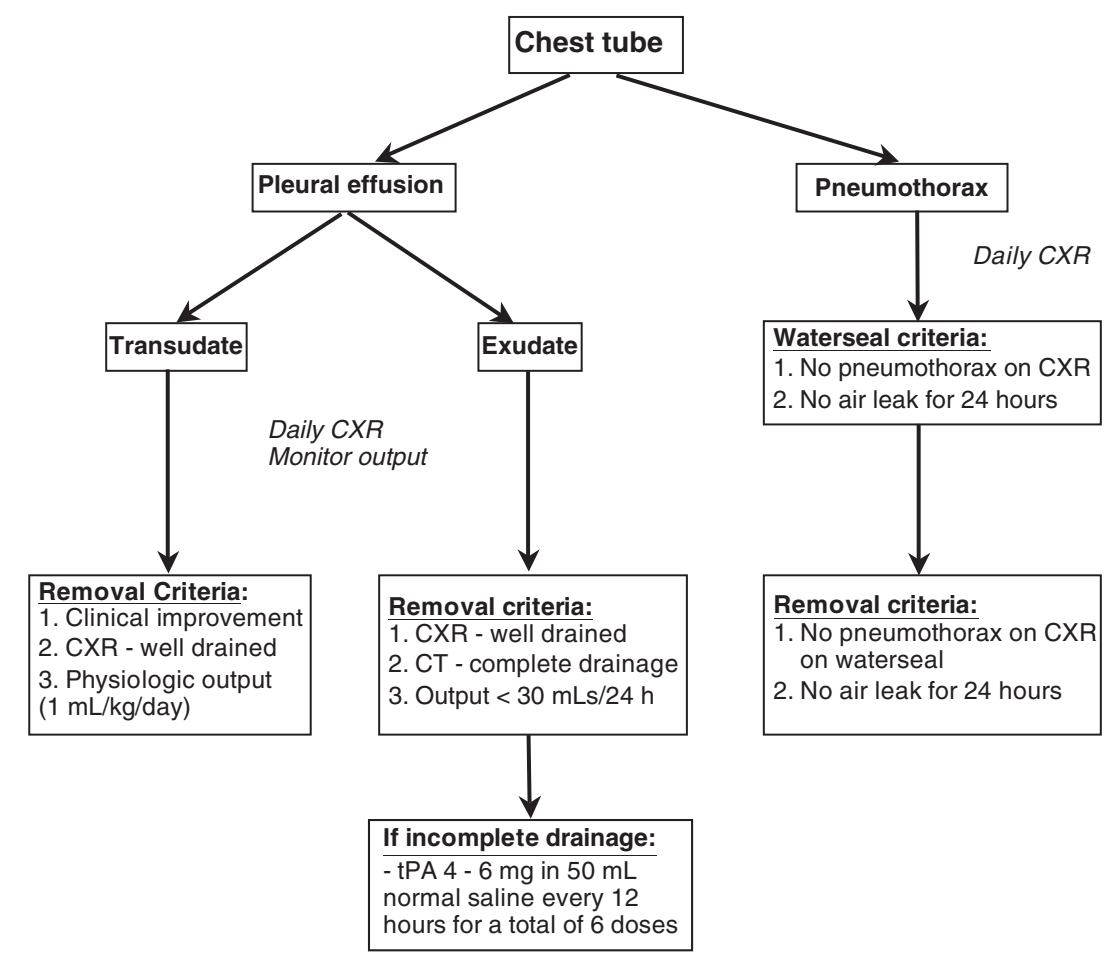

Figure 4 An algorithm for the management of chest tubes. CXR, chest X-ray; tPA, tissue plasminogen activator. 

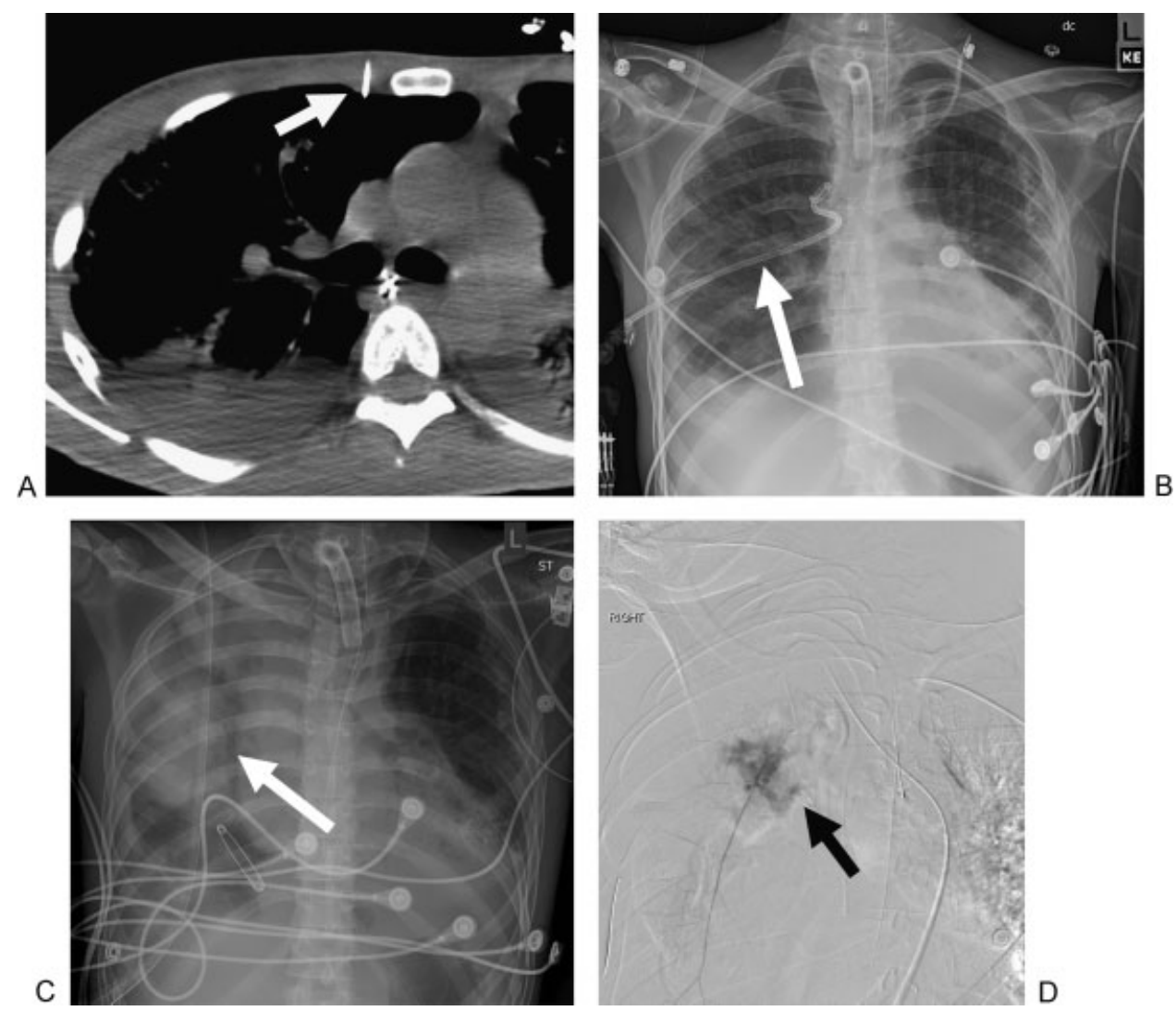

Figure 5 A 23-year-old man with bilateral pneumothoraces secondary to interstitial lung disease. (A) Axial computed tomography image of the chest at the time of the right thoracostomy demonstrates the trajectory of the drainage catheter, immediately adjacent to the internal mammary artery (arrow). (B) Frontal radiograph of the chest prior to the removal of the chest drain (arrow). (C) Frontal radiograph of the chest obtained immediately after right chest drain removal, demonstrating new right airspace and mediastinal (arrow) opacities. (D) Digital subtraction angiogram of the right internal mammary artery demonstrates active extravasation of contrast (arrow). The vessel was successfully embolized with coils.

\section{Lung Abscess}

A lung abscess usually results from aspiration of anaerobic oropharyngeal bacteria into gravity-dependent portions of the lung, most often the posterior segments of the upper lobes and the superior segments of the lower lobes. Depression of consciousness and the gag reflex due to conditions such as drug overdose, excess alcohol ingestion, neuromuscular disease, and cerebrovascular accident are all major risk factors. Esophageal conditions such as stricture, malignancy, and reflux can predispose to aspiration and subsequent abscess development. Bronchial obstruction due to malig- nancy, inflammation, or foreign body is also an important risk factor for development of lung abscess because it impairs effective clearing of aspirated oropharyngeal fluid. Other risk factors include immune deficiencies or suppression and chronic illness due to liver and kidney disease and diabetes mellitus. Consequently, lung abscesses can be classified into primary or secondary cases depending on the presence or absence of underlying conditions.

Current first-line therapy for lung abscess is antibiotic therapy directed at the suspected causative organisms, usually anaerobes or mixed aerobic and anaerobic bacteria.
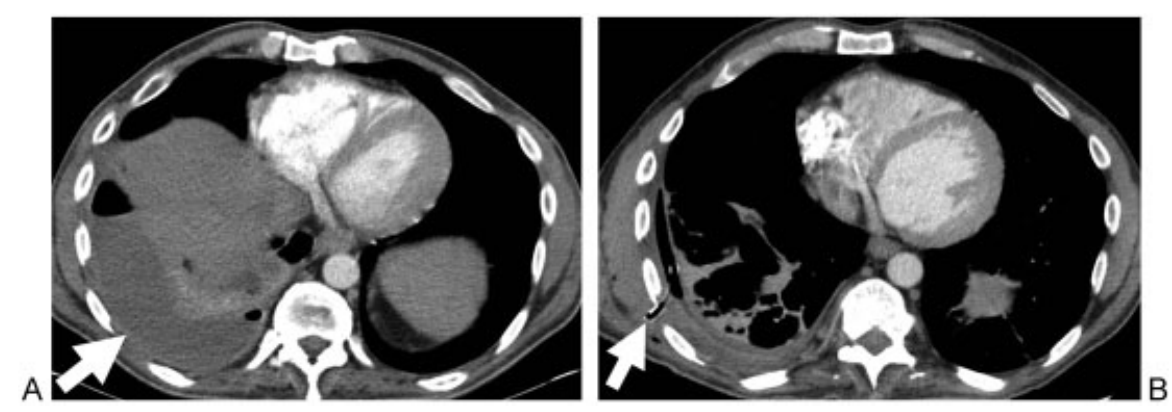

Figure 6 A 45-year-old man with fevers. (A) Axial contrast-enhanced computed tomography (CT) scan of the chest demonstrates a right empyema (arrow). (B) Axial CT scan of the chest after chest tube placement (arrow) and administration of six doses of tissue plasminogen activator. The right empyema shows near complete resolution. 

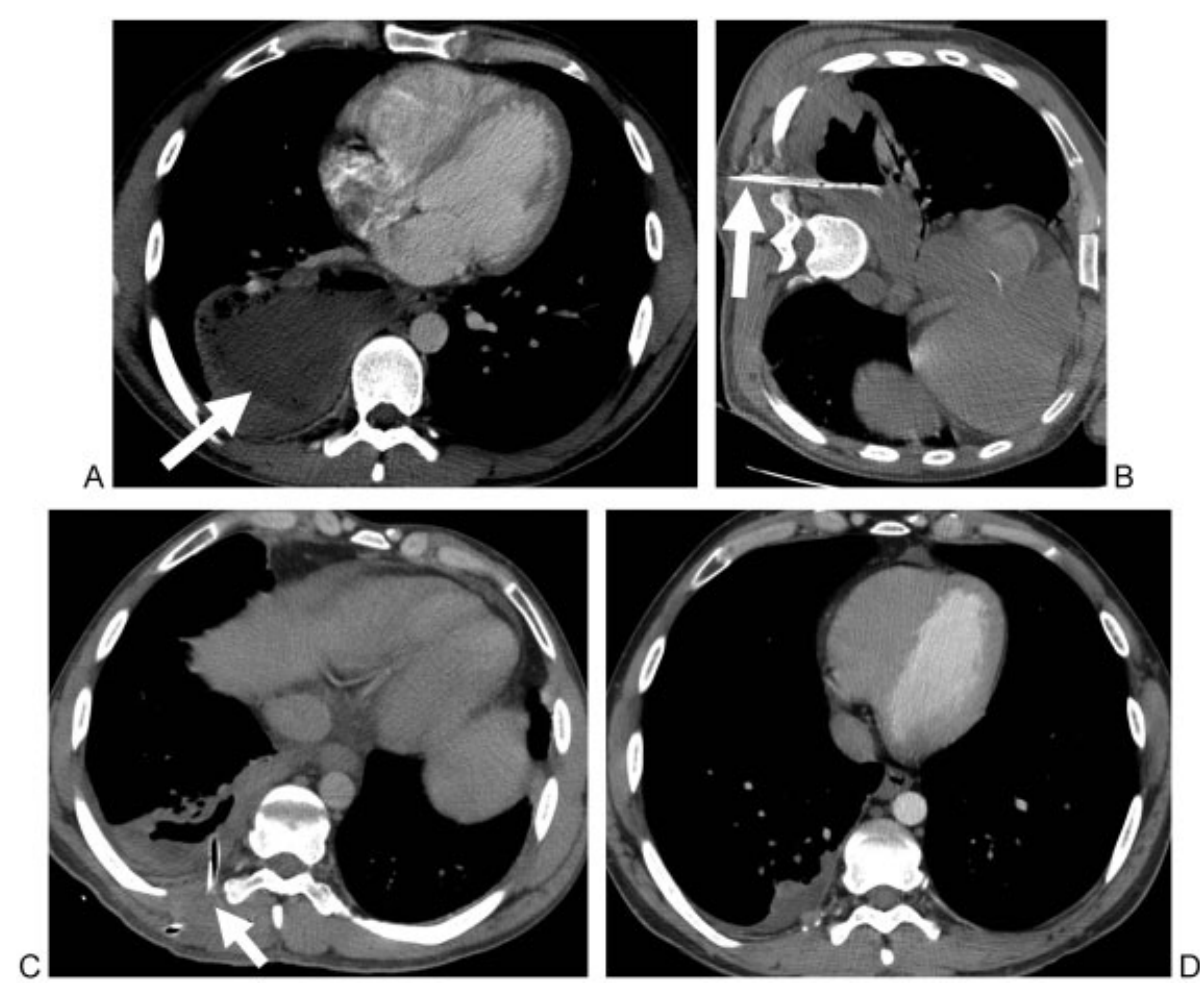

Figure 7 A 26-year-old man with a history of intravenous drug abuse who failed conservative management. (A) Axial contrast-enhanced computed tomography (CT) scan of the chest demonstrates a right lower lobe pulmonary abscess (arrow). (B) Axial CT scan of the chest with the patient in a lateral decubitus position that demonstrates the catheter (arrow) in the abscess. (C). Axial CT scan of the chest obtained 4 days later demonstrates the catheter in the abscess (arrow) and a significant decrease in the size of the abscess. (D) Axial CT scan of the chest

obtained 2 months after drainage shows residual right pleural thickening but no residual abscess.

Conservative medical management proves effective in 80 to $90 \%$ of patients with lung abscesses. ${ }^{33}$ Conservative management failure may be due to the virulence of the responsible pathogens, failure to achieve an adequate concentration of antibiotics within the abscess cavity, and/or severe underlying lung disease that may play a role in the failure of the abscess cavity to drain completely. ${ }^{34}$ Patients who display no radiographic evidence of improvement, signs of persistent sepsis, or develop complications such as hemoptysis, bronchopleural fistula, or empyema require external drainage or resection for definite treatment.

\section{Lung Parenchymal Interventions}

\section{Percutaneous Drainage Catheter Insertion}

Drainage catheter insertion is usually performed using CT guidance (-Fig. 7), but fluoroscopic or ultrasonographic guidance can also be used. When possible, normal lung should be avoided to prevent development of an infected bronchopleural fistula or pyopneumothorax and to minimize the risk of bleeding. Either tandem trocar or the Seldinger technique are effective methods for percutaneous drainage catheter. In discussing the difference between the two techniques, Erasmus et al seem to favor the Seldinger technique, stating that although direct puncture of the abscess cavity often saves time, the Seldinger technique allows more control and decreases the likelihood of complications. ${ }^{35}$ VanSonnenberg et al have also cautioned that although the trocar technique may be quite safe, using it may cause a higher incidence of undesirable trauma to the lung. ${ }^{36}$ Advocates of the trocar technique believe that it is not associated with an increased incidence of complications and is less likely to be associated with abscess contamination. ${ }^{21}$

A meta-analysis of 21 studies found that the success rate of percutaneous catheter drainage for pulmonary abscesses was $83.9 \%$, with a complication rate of $16.1 \%$; overall mortality as a complication of treatment of the abscess was $4 \%{ }^{34}$ In comparison, postoperative mortality after surgery for lung abscess has been reported to range from 11 to $16 \%{ }^{34}$ The possible complications of percutaneous drainage catheters include pneumothorax, pyopneumothorax, and bronchopleural fistula.

\section{The Mediastinum: Pathologic Conditions}

Indications for inserting percutaneous mediastinal drainage catheters include mediastinal abscess and esophageal anastomotic leaks.

\section{Mediastinal Abscess}

Mediastinal abscesses are a rare and potentially fatal condition. Mortality rates can be as high as $40 \%$ in cases when the diagnosis is not readily suspected and treatment is delayed. ${ }^{37,38}$ Common etiologies of mediastinal abscess include head and neck infections that descend into the mediastinum, trauma, and postsurgical. Mediastinal abscesses following esophagogastric surgery have a reported frequency of 8 to $21 \%$ and are the leading cause of postoperative morbidity, stricture, and repeated operation. ${ }^{39}$ 

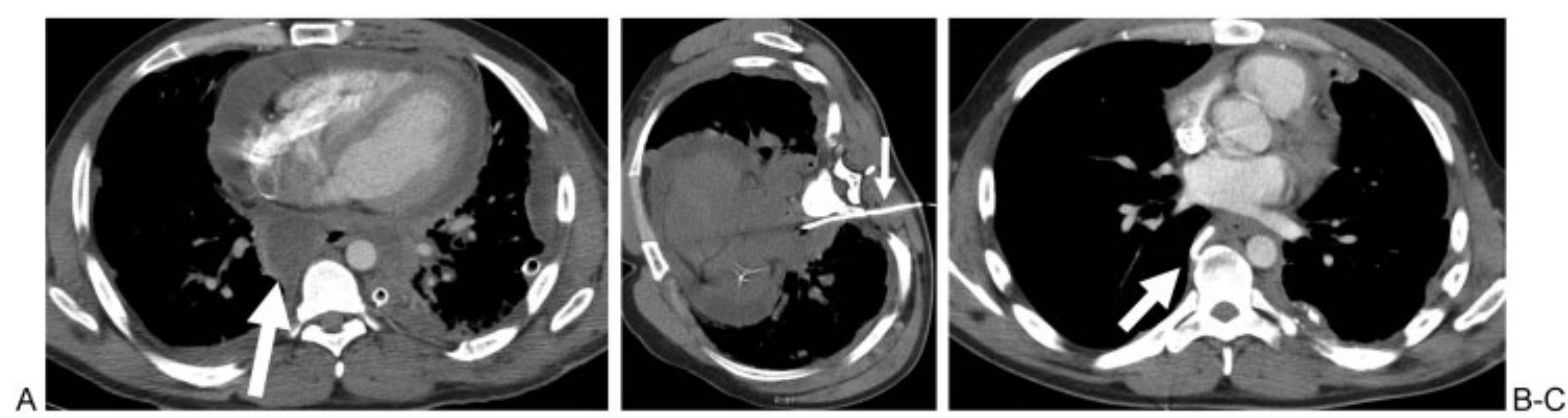

Figure 8 A 39-year-old man status post repair of an emetogenic esophageal perforation. (A) Axial contrast-enhanced computed tomography (CT) scan of the chest demonstrates a posterior mediastinal abscess (arrow). (B) Axial CT scan of the chest with the patient in a lateral decubitus position demonstrates the catheter in the abscess (arrow) while avoiding the lung and pleura. (C) Axial CT scan of the chest obtained 2 weeks after drainage demonstrates near complete resolution of the abscess (arrow).

Historically, intravenous antibiotics combined with surgical debridement (open, video assisted, or mediastinoscopically assisted drainage) have been the mainstay of treatment. ${ }^{40,41}$ Endoscopic drainage of mediastinal collections has also been described. ${ }^{42}$ Although there were several studies assessing percutaneous image-guided drainage of mediastinal abscesses in the early $1980 \mathrm{~s},{ }^{43-45}$ it is still not an established treatment option. A recent study demonstrated that percutaneous CT-guided drainage was technically successful via either trocar or Seldinger technique in a series of 25 abscess drainage attempts. ${ }^{39}$ In this report, one patient required an additional drainage procedure for a loculated posterior mediastinal abscess, but no patient required surgical debridement for failed drainage.

\section{Anastomotic Leaks}

Treatment options for anastomotic leaks after esophagectomy include surgical resection and revision of the original anastomosis, self-expanding stent placement, ${ }^{46}$ and percutaneous drainage. Percutaneous treatment of intrathoracic esophageal anastomotic leaks requires that the catheters be placed in the mediastinal collection close to the site of the anastomotic leakage to achieve adequate control of the leak. ${ }^{47}$ Six of the 23 patients in the study by Arellano et al were patients with an anastomotic leak after esophagectomy, and they reported successful drainage of the collection in all cases. ${ }^{39}$

\section{Mediastinal Interventions}

\section{Percutaneous Drainage Catheter Insertion}

Mediastinal drainage catheter insertion is usually performed under CT guidance to clearly identify the abscess and its relationship to adjacent vital structures (-Fig. 8). When inserting the catheter, there is potential for transgression of the pleura that could result in pneumothorax or in seeding the pleural cavity with infection, resulting in empyema. In some patients, it may be helpful to inject saline into the pleural cavity to separate the visceral pleura from the parietal pleura, creating a larger space in which to place catheters and thus reducing the risk of pneumothorax. ${ }^{48}$ As in other drainage procedures in the chest, mediastinal drainages can be performed using either the Seldinger or tandem trocar technique.

\section{Conclusion}

Interventional radiology plays a vital role in the management of disease processes that involve the pleura, pulmonary parenchyma, and mediastinal spaces. The fundamental principles that guide drainage procedures below the diaphragm can be applied to thoracic drainages; for many patients with pathologic conditions of the thorax, their care is centered around the technical skill and clinical expertise of the interventional radiologist.

\section{References}

1 Weldon E, Williams J. Pleural disease in the emergency department. Emerg Med Clin North Am 2012;30(2):475-499, ix-x

2 Light RW, Macgregor MI, Luchsinger PC, Ball WC Jr. Pleural effusions: the diagnostic separation of transudates and exudates. Ann Intern Med 1972;77(4):507-513

3 Heffner JE. Discriminating between transudates and exudates. Clin Chest Med 2006;27(2):241-252

4 Light RW. Parapneumonic effusions and empyema. Clin Chest Med 1985;6(1):55-62

5 Daniels CE, Ryu JH. Improving the safety of thoracentesis. Curr Opin Pulm Med 2011;17(4):232-236

6 Barnes TW, Morgenthaler TI, Olson EJ, Hesley GK, Decker PA, Ryu $\mathrm{JH}$. Sonographically guided thoracentesis and rate of pneumothorax. J Clin Ultrasound 2005;33(9):442-446

7 Raptopoulos V, Davis LM, Lee G, Umali C, Lew R, Irwin RS. Factors affecting the development of pneumothorax associated with thoracentesis. AJR Am J Roentgenol 1991;156(5):917-920

8 Grogan DR, Irwin RS, Channick R, et al. Complications associated with thoracentesis. A prospective, randomized study comparing three different methods. Arch Intern Med 1990;150(4):873-877

9 Patel PA, Ernst FR, Gunnarsson CL. Ultrasonography guidance reduces complications and costs associated with thoracentesis procedures. J Clin Ultrasound 2012;40(3):135-141

10 Duszak R Jr, Chatterjee AR, Schneider DA. National fluid shifts: fifteen-year trends in paracentesis and thoracentesis procedures. J Am Coll Radiol 2010;7(11):859-864

11 Gordon CE, Feller-Kopman D, Balk EM, Smetana GW. Pneumothorax following thoracentesis: a systematic review and metaanalysis. Arch Intern Med 2010;170(4):332-339

12 Heidecker J, Huggins JT, Sahn SA, Doelken P. Pathophysiology of pneumothorax following ultrasound-guided thoracentesis. Chest 2006;130(4):1173-1184

13 Feller-Kopman D, Berkowitz D, Boiselle P, Ernst A. Large-volume thoracentesis and the risk of reexpansion pulmonary edema. Ann Thorac Surg 2007;84(5):1656-1661 
14 Mahfood S, Hix WR, Aaron BL, Blaes P, Watson DC. Reexpansion pulmonary edema. Ann Thorac Surg 1988;45(3):340-345

15 Long SS, Johnson PT, Fishman EK. Intercostal artery pseudoaneurysm due to thoracentesis: diagnosis with three-dimensional computed tomographic angiography. J Comput Assist Tomogr 2012; 36(1):100-102

16 Yacovone ML, Kartan R, Bautista M. Intercostal artery laceration following thoracentesis. Respir Care 2010;55(11):1495-1498

17 Dewhurst C, O'Neill S, O'Regan K, Maher M. Demonstration of the course of the posterior intercostal artery on CT angiography: relevance to interventional radiology procedures in the chest. Diagn Interv Radiol 2012;18(2):221-224

18 Yoneyama H, Arahata M, Temaru R, Ishizaka S, Minami S. Evaluation of the risk of intercostal artery laceration during thoracentesis in elderly patients by using 3D-CT angiography. Intern Med 2010;49(4):289-292

19 Cervini P, Hesley GK, Thompson RL, Sampathkumar P, Knudsen JM. Incidence of infectious complications after an ultrasound-guided intervention. AJR Am J Roentgenol 2010;195(4):846-850

20 Van Meter ME, McKee KY, Kohlwes RJ. Efficacy and safety of tunneled pleural catheters in adults with malignant pleural effusions: a systematic review. J Gen Intern Med 2011;26(1):70-76

21 Silverman SG, Mueller PR, Saini S, et al. Thoracic empyema: management with image-guided catheter drainage. Radiology 1988;169(1):5-9

22 Havelock T, Teoh R, Laws D, Gleeson F; BTS Pleural Disease Guideline Group. Pleural procedures and thoracic ultrasound: British Thoracic Society Pleural Disease Guideline 2010. Thorax 2010;65(Suppl 2):ii61-ii76

23 Bell RL, Ovadia P, Abdullah F, Spector S, Rabinovici R. Chest tube removal: end-inspiration or end-expiration? J Trauma 2001; 50(4):674-677

24 Eisenberg RL, Khabbaz KR. Are chest radiographs routinely indicated after chest tube removal following cardiac surgery? AJR Am J Roentgenol 2011;197(1):122-124

25 Goodman MD, Huber NL, Johannigman JA, Pritts TA. Omission of routine chest $\mathrm{X}$-ray after chest tube removal is safe in selected trauma patients. Am J Surg 2010;199(2):199-203

26 Gervais DA, Levis DA, Hahn PF, Uppot RN, Arellano RS, Mueller PR. Adjunctive intrapleural tissue plasminogen activator administered via chest tubes placed with imaging guidance: effectiveness and risk for hemorrhage. Radiology 2008;246(3):956-963

27 Lee KS, Im JG, Kim YH, Hwang SH, Bae WK, Lee BH. Treatment of thoracic multiloculated empyemas with intracavitary urokinase: a prospective study. Radiology 1991;179(3):771-775

28 Park CS, Chung WM, Lim MK, Cho CH, Suh CH, Chung WK. Transcatheter instillation of urokinase into loculated pleural effusion: analysis of treatment effect. AJR Am J Roentgenol 1996;167(3): 649-652

29 Misthos P, Sepsas E, Konstantinou M, Athanassiadi K, Skottis I, Lioulias A. Early use of intrapleural fibrinolytics in the management of postpneumonic empyema. A prospective study. Eur J Cardiothorac Surg 2005;28(4):599-603

30 Tokuda Y, Matsushima D, Stein GH, Miyagi S. Intrapleural fibrinolytic agents for empyema and complicated parapneumonic effusions: a meta-analysis. Chest 2006;129(3):783-790

31 Maskell NA, Davies CW, Nunn AJ, et al; First Multicenter Intrapleural Sepsis Trial (MIST1) Group. U.K. Controlled trial of intra- pleural streptokinase for pleural infection. N Engl J Med 2005; 352(9):865-874

32 Rahman NM, Maskell NA, West A, et al. Intrapleural use of tissue plasminogen activator and DNase in pleural infection. $\mathrm{N}$ Engl $\mathrm{J}$ Med 2011;365(6):518-526

33 Klein JS, Schultz S, Heffner JE. Interventional radiology of the chest: image-guided percutaneous drainage of pleural effusions, lung abscess, and pneumothorax. AJR Am J Roentgenol 1995; 164(3):581-588

34 Wali SO. An update on the drainage of pyogenic lung abscesses. Ann Thorac Med 2012;7(1):3-7

35 Erasmus JJ, McAdams HP, Rossi S, Kelley MJ. Percutaneous management of intrapulmonary air and fluid collections. Radiol Clin North Am 2000;38(2):385-393

36 vanSonnenberg E, D'Agostino HB, Casola G, Wittich GR, Varney RR, Harker C. Lung abscess: CT-guided drainage. Radiology 1991;178 (2):347-351

37 Isaacs LM, Kotton B, Peralta MM Jr, et al. Fatal mediastinal abscess from upper respiratory infection. Ear Nose Throat J 1993;72(9): 620-622, 624-626, 631

38 Misthos P, Katsaragakis S, Kakaris S, Theodorou D, Skottis I. Descending necrotizing anterior mediastinitis: analysis of survival and surgical treatment modalities. J Oral Maxillofac Surg 2007; 65(4):635-639

39 Arellano RS, Gervais DA, Mueller PR. Computed tomographyguided drainage of mediastinal abscesses: clinical experience with 23 patients. J Vasc Interv Radiol 2011;22(5):673-677

40 Chen KC, Chen JS, Kuo SW, et al. Descending necrotizing mediastinitis: a 10-year surgical experience in a single institution. J Thorac Cardiovasc Surg 2008;136(1):191-198

41 Gorlitzer M, Grabenwoeger M, Meinhart J, et al. Descending necrotizing mediastinitis treated with rapid sternotomy followed by vacuum-assisted therapy. Ann Thorac Surg 2007; 83(2):393-396

42 Jorgensen JO, Hunt DR. Endoscopic drainage of esophageal suture line leaks. Am J Surg 1993;165(3):362-364

43 Gobien RP, Stanley JH, Gobien BS, Vujic I, Pass HI. Percutaneous catheter aspiration and drainage of suspected mediastinal abscesses. Radiology 1984;151(1):69-71

44 Karlson KB, Martin EC, Fankuchen EI, et al. Non-surgical drainage of intra-abdominal and mediastinal abscesses: a report of twelve cases. Cardiovasc Intervent Radiol 1981;4(3):170-176

45 Mandel SR, Boyd D, Jaques PF, Mandell V, Staab EV. Drainage of hepatic, intraabdominal, and mediastinal abscesses guided by computerized axial tomography. Successful alternative to open drainage. Am J Surg 1983;145(1):120-125

46 van Boeckel PG, Sijbring A, Vleggaar FP, Siersema PD. Systematic review: temporary stent placement for benign rupture or anastomotic leak of the oesophagus. Aliment Pharmacol Ther 2011; 33(12):1292-1301

47 Maher MM, Lucey BC, Boland G, Gervais DA, Mueller PR. The role of interventional radiology in the treatment of mediastinal collections caused by esophageal anastomotic leaks. AJR Am J Roentgenol 2002;178(3):649-653

48 Langen HJ, Klose KC, Keulers P, Adam G, Jochims M, Günther RW. Artificial widening of the mediastinum to gain access for extrapleural biopsy: clinical results. Radiology 1995;196(3): 703-706 